\title{
A RADIO COLORING OF A HYPERCUBE
}

\author{
OPHIR FRIEDER ${ }^{\mathrm{a}, *}$, FRANK HARARY ${ }^{\mathrm{b}}$ and PENG-JUN WAN ${ }^{\mathrm{a}}$ \\ a Illinois Institute of Technology, Chicago, IL 60616; \\ ${ }^{\mathrm{b}}$ New Mexico State University, \\ Las Cruces, NM 88003
}

(Received 23 May 2001; In final form 26 May 2001)

\begin{abstract}
A radio coloring of a graph $G$ is an assignment of nonnegative integers to its nodes so that each pair of adjacent nodes have color numbers that differ by at least two, and any pair of nodes at distance 2 have different colors. Every graph has a radio coloring by simply assigning the odd integers $1,3,5, \ldots$, but there is then a big difference between the smallest and largest colors. We define the span of a radio coloring of $G$ as one plus the difference between the smallest and largest colors. We study radio colorings of a hypercube with the objective of finding such a coloring with minimum span. We develop a formulation for what we believe is the complete solution to this question in the form of a conjecture.
\end{abstract}

Keywords: Hypercubes; Radio coloring; Hamming code

C. R. Categories: G.2.2

\section{INTRODUCTION}

Let $V_{n}$ denote the $n$-dimensional vector space over $G F(2)$, the Galois Field of order 2 . Note that each vector in $V_{n}$ corresponds to a binary representation of some integer between 0 and $2^{n}-1$, and vice versa. Let $x_{n-1} \cdots x_{1} x_{0}$ be the binary representation of $k$ for $0 \leq k \leq 2^{n}-1$. Then, we define

$$
B_{n}(k)=\left(x_{n-1}, \ldots, x_{1}, x_{0}\right)
$$

For any $u, v \in V_{n}$, as usual in graph theory, we write $d(u, v)$ to denote the distance between $u$ and $v$ in the hypercube $Q_{n}$. This is the smallest length of a path between $u$ and $v$, and so it is the number of positions where $u$ and $v$ differ; see the book [2].

The $n$-dimensional hypercube $Q_{n}$ is a graph over $V_{n}$ in which two nodes are adjacent if they agree in all but one position. A vertex coloring of a graph by nonnegative integers is called a radio coloring [3] if any pair of adjacent nodes have color numbers that differ by at least two, and any pair of nodes at distance 2 have different colors. The span of any radio coloring is defined as one plus the difference between the largest color number and the smallest color number. Our objective is to investigate a radio coloring of a hypercube

\footnotetext{
*Corresponding author. E-mail: ophir@cs.iit.edu
} 
with minimal span. Without loss of generality, we restrict the smallest color number to be zero.

\section{COLORING VERSUS CODING}

Consider any radio coloring of $Q_{n}$ given by a function $c: V_{n} \mapsto Z^{+}$. Let

$$
r=\left\lceil\log _{2}\left(1+\max \left\{c(v): v \in V_{n}\right\}\right)\right\rceil .
$$

Then, each color can be represented by a $r$-bit binary number. So the vertex coloring $c$ gives rise to a binary code in which each code-word is a concatenation of some $v \in V_{n}$ and $B_{r}(c(v))$.

On the other hand, any linear binary code also induces a radio coloring of a hypercube. Let $C$ be any $[n+r, n]$-code in which the leftmost $n$ bits are the data bits and the remaining $r$ bits are the check bits. Then, for each vertex $v \in V_{n}$, we assign the color whose binary representation is the check bits of $v$ in the code $C$.

Of particular interest is the single-error correcting linear code. One well-known singleerror correcting linear code is the Hamming code [1]. The Hamming code is optimal in the sense that it uses the smallest number of check bits. If the number of data bits is $n$, the Hamming code uses $f(n)$ check bits where

$$
f(n)=\min \left\{r: n+r+1 \leq 2^{r}\right\} .
$$

For example,

$$
\begin{aligned}
f(1) & =2, \\
f(2) & =f(3)=f(4)=3, \\
f(5) & =\cdots=f(11)=4, \\
f(12) & =\cdots=f(26)=5, \\
f(27) & =\cdots=f(57)=6 .
\end{aligned}
$$

A single-error correcting linear code has distance at least three. Thus, the coloring induced by such a code has the following property:

1. for any pair of nodes at distance 2, their colors are different;

2. for any pair of adjacent nodes, the binary representations of their colors differ in at least two positions.

In general, however, the single-error correcting code may not yield a radio coloring. Note that the second property does not necessarily imply that the difference between the values of the colors assigned to adjacent nodes is greater than one. For example, in $Q_{4}$ the color numbers 7 (0111) and 8 (1000) vary in all four bits but have a color number difference of only one. The following lemma shows that the binary representations of two consecutive numbers can differ in many positions.

LEMMA 1. For any $0 \leq k<2^{r}-1$, let $i$ be the position (counting from right to left) of the rightmost 0 in $B_{r}(k)$. Then

$$
B_{r}(k+1)-B_{r}(k)=B_{r}\left(2^{i}-1\right) .
$$


Therefore, the single-error correcting code cannot give rise directly to a radio coloring. Nevertheless, it provides us with insight into the design of a node coloring approach. We now provide a coloring whose corresponding code also uses $f(n)$ check bits for $n$ data bits.

\section{A RADIO COLORING OF $Q_{n}$}

Let $S$ be the set of all positive integers excluding $\left\{2^{i}-1: i \geq 1\right\}$, i.e.,

$$
S=\{2,4,5,6,8,9,10,11,12,13,14,16, \ldots\}
$$

For each $i \geq 1$, let $s_{i}$ denote the $i$ th smallest number in $S$. Then, for all $i \geq 1$,

$$
\begin{aligned}
2^{f(i)-1} & \leq s_{i}<2^{f(i)}-1, \\
s_{2^{i}-i} & =2^{i} .
\end{aligned}
$$

For any $n \geq 1$, we define $A(n)$ to be the $n \times f(n)$ matrix in which the $i$ th row from the bottom is $B_{f(n)}\left(s_{i}\right)$, the $f(n)$-dimensional vector corresponding to the $i$ th number in $S$. For example,

$$
\begin{aligned}
A(3) & =\left(\begin{array}{lll}
1 & 0 & 1 \\
1 & 0 & 0 \\
0 & 1 & 0
\end{array}\right), \\
A(4) & =\left(\begin{array}{lll}
1 & 1 & 0 \\
1 & 0 & 1 \\
1 & 0 & 0 \\
0 & 1 & 0
\end{array}\right), \\
A(5) & =\left(\begin{array}{llll}
1 & 0 & 0 & 0 \\
0 & 1 & 1 & 0 \\
0 & 1 & 0 & 1 \\
0 & 1 & 0 & 0 \\
0 & 0 & 1 & 0
\end{array}\right) .
\end{aligned}
$$

Now we are ready to describe our node coloring. For each (row) vector $v \in V_{n}$, its color is given by

$$
c(v)=B_{f(n)}^{-1}(v A(n)),
$$

i.e., the number whose binary representation corresponds to the vector $v A(n)$. For example, when $n=3$,

$$
\begin{aligned}
& c(000)=0, \quad c(001)=2, \quad c(010)=4, \quad c(011)=6, \\
& c(100)=5, \quad c(101)=7, \quad c(110)=1, \quad c(111)=3 .
\end{aligned}
$$


When $n=4$,

$$
\begin{array}{llll}
c(0000)=0, & c(0001)=2, & c(0010)=4, & c(0011)=6, \\
c(0100)=5, & c(0101)=7, & c(0110)=1, & c(0111)=3, \\
c(1000)=6, & c(1001)=4, & c(1010)=2, & c(1011)=0, \\
c(1100)=3, & c(1101)=1, & c(1110)=7, & c(1111)=5 .
\end{array}
$$

The next lemma proves that the above node coloring is indeed a radio coloring.

LEMMA 2. Let $u$ and $v$ be any two nodes of $Q_{n}$.

(1) If $d(u, v)=1$, then $|c(u)-c(v)| \geq 2$.

(2) If $d(u, v)=2$, then $c(u) \neq c(v)$.

Proof. We first prove (1). Suppose that nodes $u$ and $v$ differ only at the position $i$ (counting from right to left) for some $1 \leq i \leq f(n)$. Then

$$
\begin{aligned}
B_{f(n)}(c(u))-B_{f(n)}(c(v)) & =u A(n)-v A(n) \\
& =(u-v) A(n) \\
& =B_{f(n)}\left(s_{i}\right) .
\end{aligned}
$$

As $B_{f(n)}\left(s_{i}\right) \neq 0$, we have $c(u) \neq c(v)$. Furthermore, as $B_{f(n)}\left(s_{i}\right) \neq 2^{k}-1$ for any $k>0$, we have $|c(u)-c(v)| \neq 1$ from Lemma 1. Therefore $|c(u)-c(v)| \geq 2$.

Now we prove (2). Suppose that $u$ and $v$ differ only at the positions $i$ and $j$ (counting from right to left) for some $1 \leq i<j \leq f(n)$. Then

$$
\begin{aligned}
B_{f(n)}(c(u))-B_{f(n)}(c(v)) & =(u-v) A(n) \\
& =B_{f(n)}\left(s_{i}\right)+B_{f(n)}\left(s_{j}\right) \\
& =B_{f(n)}\left(s_{i}\right)-B_{f(n)}\left(s_{j}\right) \\
& \neq 0 .
\end{aligned}
$$

This implies that $c(u) \neq c(v)$.

As each color is a $f(n)$-bit binary number, at most $2^{f(n)}$ colors are used by the above node coloring. Now we show that exactly $2^{f(n)}$ colors are used when $n>2$. The proof is completed in two steps. In the first step, we show that for any $0 \leq i \leq f(n)-1$ the color $2^{i}$ is assigned to some node. We consider two cases.

Case 1: $1 \leq i \leq f(n)-1$. Since $B_{n}\left(2^{2^{i}-i-1}\right)$ have zeros in all positions except position $2^{i}-i$ (counting from right),

$$
B_{n}\left(2^{2^{i}-i-1}\right) A(n)=B_{f(n)}\left(s_{2^{i}-i}\right)=B_{f(n)}\left(2^{i}\right) .
$$

This implies that the node $B_{n}\left(2^{2^{i}-i-1}\right)$ is assigned color $2^{i}$. 
Case 2: $\quad i=0$. Since $B_{n}(6)$ has zeros in all positions except position 2 and 3 (counting from right),

$$
\begin{aligned}
B_{n}(6) A(n) & =B_{f(n)}\left(s_{2}\right)+B_{f(n)}\left(s_{3}\right) \\
& =B_{f(n)}(4)+B_{f(n)}(5)=B_{f(n)}\left(2^{0}\right) .
\end{aligned}
$$

This implies that the node $B_{n}(6)$ is assigned color $2^{0}$.

In the second step, we show that for any $k$ with $0 \leq k \leq 2^{f(n)}-1$, the color number $k$ is assigned to some node. Define $H(n)$ to be the $f(n) \times n$ matrix in which the bottom row is $B_{n}(6)$, and for any $2 \leq i \leq f(n)$, the $i$ th row from the bottom is $B_{n}\left(2^{2^{i-1}-i-2}\right)$. For example,

$$
H(4)=\left(\begin{array}{llll}
0 & 0 & 1 & 0 \\
0 & 0 & 0 & 1 \\
0 & 1 & 1 & 0
\end{array}\right)
$$

Then, for any $1 \leq i \leq f(n)$ the $i$ th row from the bottom of $H(n)$ times $A(n)$ is equal to $B_{f(n)}\left(2^{i-1}\right)$. So the matrix $H(n) A(n)$ is a $f(n) \times f(n)$ identity matrix, which means that $H(n)$ is a left inverse of $A(n)$. Thus, for any $0 \leq k \leq 2^{f(n)}-1$, the color assigned to vertex $B_{f(n)}(k) H(n)$ is

$$
B_{f(n)}^{-1}\left(B_{f(n)}(k) H(n) A(n)\right)=B_{f(n)}^{-1}\left(B_{f(n)}(k)\right)=k
$$

Then, for any color $0 \leq k \leq 2^{f(n)}-1$, it is assigned to node $B_{f(n)}(k) H(n)$.

The above node coloring is also node-balanced in the sense that each color is shared by the same number of nodes. In fact, this is true when $n=2$. Assume that $n>2$. We show that the matrix $A(n)$ always has a full rank of $f(n)$. It is easy to verify that $f(n) \leq n$. The matrix $A(n)$ contains the row vectors $B_{f(n)}\left(2^{i}\right)$ for $1 \leq i \leq f(n)-1$. Notice that the sum of the second row from the bottom and the third row from the bottom gives rise to another row vector $B_{f(n)}\left(2^{0}\right)$. As the $f(n)$ row vectors $B_{f(n)}\left(2^{i}\right)$ for $0 \leq i \leq f(n)-1$ are independent, the rank of the matrix $A(n)$ is $f(n)$ when $n>2$. Therefore, the dimension of the null space of $A(n)$ is equal to $n-f(n)$. Since for any $0 \leq k \leq 2^{f(n)}-1$ the equation

$$
v A(n)=B_{f(n)}(k)
$$

has at least a solution, the number of solutions is thus exactly $2^{n-f(n)}$. This implies that each color is assigned to $2^{n-f(n)}$ nodes.

\section{DISCUSSION}

An obvious lower bound on the minimum span of any radio coloring of the $n$-cube is $n+2$. In fact, consider the node which is assigned color 0 . Then, none of its $n$ neighbors can be assigned color 0 or 1 . In addition, these $n$ neighbors must all have distinct colors. Thus, the largest color used by any of these $n$ neighbors must be at least $n+1$.

The radio coloring given in the previous section is not optimal when $n \leq 3$. In fact, when $n=2$, the following coloring is better (see Fig. 1(a)):

$$
c(00)=0, \quad c(01)=3, \quad c(10)=4, \quad c(11)=1 .
$$




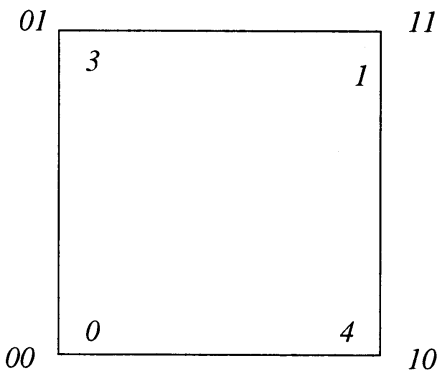

(a)

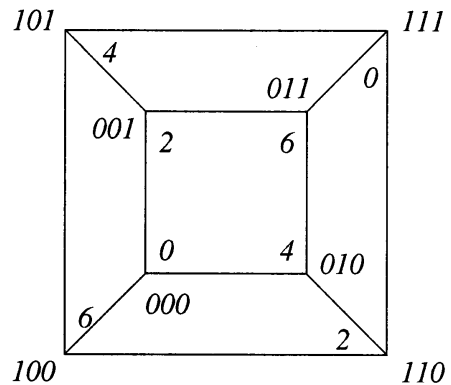

(b)

FIGURE 1 The optimal radio colorings when $n=2,3$. They differ from those in Section 3 .

When $n=3$, the following coloring is better (see Fig. 1(b)):

$$
\begin{array}{ll}
c(000)=c(111)=0, & c(001)=c(110)=2, \\
c(010)=c(101)=4, & c(011)=c(100)=6 .
\end{array}
$$

When $n \geq 4$, we make the following statement.

CONJECTURE The radio coloring of a hypercube given in Section 3 is optimal for minimizing the span when $n \geq 4$.

\section{References}

[1] Roman, S. Coding and Information Theory, Springer-Verlag, New York, (1992).

[2] Harary, F. (1969) Graph Theory, Addison-Wesley, Reading, MA.

[3] Harary, F. and Plantholt, M., Graphs whose radio coloring number equals the number of nodes, Graph Colouring and Applications CRM Proc. Lecture Notes, Amer. Math. Soc. 23, 99-100, (1999). 
Copyright of International Journal of Computer Mathematics is the property of Taylor \& Francis Ltd and its content may not be copied or emailed to multiple sites or posted to a listserv without the copyright holder's express written permission. However, users may print, download, or email articles for individual use. 